\section{Cost Savings and Burden of an Intravascular Line Tip Culture Screening Policy}

To the Editor-Intravascular-catheter-mediated bacteremia is responsible for $>60 \%$ of hospital infections. ${ }^{1}$ The intravascular catheter culture is necessary for the diagnosis of suspected phlebitis and catheter-associated bacteremia. Catheter-tip culture is a test that has a low predictive value for the diagnosis, and its importance is questionable $\left(10 \%-14 \%\right.$ bacteremia) ${ }^{2}$

One way of balancing this epidemiological issue and avoiding unnecessary culture requests and the administration of antibiotics only because of a positive catheter-tip culture would be to consider catheter-tip storage. Storage allows the culture to be performed later when there is a positive blood culture to aid in the diagnosis of a central catheter-related bloodstream infection.

Generally, physicians request the culture of all catheters, even those without suspicion of bacteremia or phlebitis. In 1992, Widmer et $\mathrm{al}^{3}$ evaluated the clinical importance of the 157 catheters that were cultured; $96 \%$ had no clinical impact. The catheter cooling method consists of cooling the central catheter tip and culturing it only for those cases with a positive peripheral blood culture. ${ }^{4}$ In addition, a recent randomized study showed increased predictive value and decreased cost compared to direct processing. ${ }^{2}$ The use of the catheter-tip cooling technique in the United Kingdom resulted in a $75 \%$ reduction in catheter cultures, representing a $42 \%$ reduction in expenditure, as well as clinical acceptance by the medical staff. ${ }^{5}$

The objective of this study was to evaluate the savings associated with the implementation of a central-catheter-tip cooling protocol for tips forwarded to the microbiology laboratory.

The study was conducted at Hospital Santa Casa de Curitiba and at Hospital Universitário Evangélico de Curitiba (HUEC) between September 2014 and October 2015. Hospital Santa Casa is a 217-bed university hospital with a specialization in cardiac surgery and heart transplantation; HUEC has 548 beds and is a national center for several specialties. During the study period, a catheter-tip storage protocol through cooling was implemented to reduce direct costs (processing and culture) and indirect, nonmeasurable costs (possible treatments by contamination or colonization). The routine consisted of storing all the central venous catheter tips in a refrigerator $\left(4^{\circ} \mathrm{C}\right)$ without immediately performing the culture and susceptibility tests. The catheter was cultured only if there had been a culture in the prior 7 days or if there had been a positive blood culture $\geq 7$ days after receipt of the catheter. If, 7 days after receiving the catheter, there was no positive culture, the material was discarded.
Intravascular catheters were cultured using the rolling technique (ie, the Maki technique) on blood agar at $37^{\circ} \mathrm{C}$ for 48 hours. Growth $>15$ colonies was considered significant. The microorganisms found were cultured and identified using standard laboratory methods.

To effectively treat the patient, physicians could request the culture of any catheter for up to 7 days, even when not associated with bacteremia because phlebitis can manifest without bacteremia.

Data regarding the number of catheters received, stored, and cultured, as well as data related to blood cultures received in the same period, were stored in the microbiology section of the database.

After 6 months of protocol establishment, the culture data and laboratorial costs were analyzed. These costs were estimated from the reagents used.

During the study period, 536 catheter tips were received for culture. Following the catheter screening policy, only $39.4 \%$ were processed. Of the catheters processed, $39.8 \%$ had negative culture and $60.2 \%$ were positive, and the most prevalent microorganism was coagulase-negative Staphylococcus (CNS) (Table 1).

Regarding the blood cultures of processed catheters, the most prevalent microorganism identified was CNS (48\% incidence) followed by Candida ( $9 \%$ incidence).

Concordance refers to the presence of the same microorganism present in the blood culture and catheter, thus indicating that bacteremia was caused by the catheter.

тAвLE 1. Results for Central-Venous-Catheter-Tip Cultures and Blood Cultures of Catheters That Were Processed After Implementation of a Central-Venous-Catheter-Tip Screening Protocol

\begin{tabular}{lrr}
\hline Variable & No. & \multicolumn{1}{c}{$\%$} \\
\hline Total CVC in the period & 449 & 100.0 \\
Discarded & 276 & 61.5 \\
Processed & 173 & 38.5 \\
Negative & 56 & 32.4 \\
Coagulase-negative Staphylococci & 54 & 31.2 \\
Candida spp. & 6 & 3.5 \\
Staphylococcus aureus & 18 & 10.4 \\
Gram-negative bacilli & 36 & 20.8 \\
Enterococcus spp. & 3 & 1.7 \\
Blood culture of processed catheters & & \\
Coagulase-negative Staphylococci & 61 & 45.9 \\
Candida spp. & 9 & 6.8 \\
Klebsiella spp. & 13 & 9.8 \\
Enterobacter spp. & 11 & 8.3 \\
Acinetobacter spp. & 7 & 5.3 \\
S. aureus & 21 & 2.3 \\
Pseudomonas aeruginosa & 3 & 6.0 \\
Other & 8 & \\
\hline
\end{tabular}

NOTE. CVC, central venous catheter. 
In this study, concordance after catheter screening was only $20.4 \%$.

The catheter screening policy provided a reduction of $27.50 \%$ in culture requests. Considering that the processing value of a positive catheter tip is US $\$ 13.62$ and of a negative catheter tip is US\$2.03, with an estimated annual savings of US $\$ 4,207.06$. Considering the hospital occupation rate, this would generate a savings of US $\$ 5.49$ per bed per year.

According to the Centers for Disease Prevention (CDC), the catheter-tip culture should only be performed when catheterrelated bacteremia is suspected. ${ }^{5}$ After 6 months of adherence to the catheter screening protocol, $43.68 \%$ of all catheter tips received was processed; catheter tips were processed if there had been a culture in the prior 7 days, a positive blood culture after 7 days of catheter arrival, or at the physician's request. However, $39.4 \%$ of the processed catheters had negative cultures, and $60.2 \%$ had positive cultures.

The concordance between culture catheter and blood culture was $20.4 \%$; thus, the percentage of catheters presenting the microorganism causing bacteremia is small. In a similar analysis, Ekkelenkamp et al ${ }^{6}$ concluded that only $5 \%-10 \%$ of the analyzed catheters are in concordance.

Regarding the economic analysis, the catheter screening policy provided a $74 \%$ reduction in material expenditures and human resources; Bouza et al ${ }^{4}$ reached $69 \%$ savings in a similar study. Brazil has 6,657 hospitals, $30 \%$ of which are public, and the savings for the public health system with the implementation of the catheter screening policy would be an estimated US $\$ 2,483,513.68$ annually.

In summary, a catheter screening protocol is an efficient way to reduce costs and avoid unnecessary use of antibiotics without detracting from patient care.

\section{ACKNOWLEDGMENTS}

We thank the ICU staff for assistance. Felipe F. Tuon is a member of the National Council for Scientific and Technological Development (CNPQ).

Financial support: No financial support was provided relevant to this article. Potential conflicts of interest: All authors report no conflicts of interest relevant to this article.

\section{Felipe Francisco Tuon, PhD; ${ }^{1,2}$ Sarah Pacher, MS ${ }^{1}$ Laryssa Gonçalves Moreira, MS; ${ }^{3}$ Guilherme Becker, BSc PSc; Juliette Cielinski, BSc PSc}

Affiliations: 1. Department of Medicine, School of Health and Biosciences, Pontifícia Universidade Católica do Paraná, Curitiba, PR, Brazil; 2. Division of Infectious Diseases, Hospital de Clínicas da Universidade Federal do Paraná. Curitiba, PR, Brazil; 3. Faculdade Evangélica do Paraná (FEPAR), Curitiba, PR, Brazil; 4. Laboratory of Microbiology, Hospital Santa Casa de Curitiba, Curitiba, PR, Brazil; 5. Laboratory of Microbiology, Hospital Universitário Evangélico de Curitiba, Curitiba, PR, Brazil.

Address correspondence to Felipe F. Tuon, PhD, Departamento de Saúde Comunitária, $7^{\circ}$. Andar, R. Padre Camargo, 280 - Alto da Glória, Curitiba - PR, 80060-240 Brazil (tuon@ufpr.br).
Infect Control Hosp Epidemiol 2017;38:1010-1011

(C) 2017 by The Society for Healthcare Epidemiology of America. All rights reserved. 0899-823X/2017/3808-0021. DOI: 10.1017/ice.2017.103

\section{REFERENCES}

1. Bouza E, San Juan R, Munoz P, et al. A European perspective on intravascular catheter-related infections: report on the microbiology workload, aetiology and antimicrobial susceptibility (ESGNI-005 Study). Clin Microbiol Infect 2004;10:838-842.

2. Colston J, Batchelor B, Bowler IC. Cost savings and clinical acceptability of an intravascular line tip culture triage policy. J Hosp Infect 2013;84:77-80.

3. Widmer AF, Nettleman M, Flint K, Wenzel RP. The clinical impact of culturing central venous catheters. A prospective study. Arch Intern Med 1992;152:1299-1302.

4. Bouza E, Guembe M, Gomez H, Martin-Rabadan P, Rivera M, Alcala L. Are central venous catheter tip cultures reliable after 6-day refrigeration? Diagn Microbiol Infect Dis 2009;64:241-246.

5. Perez-Parra A, Guembe M, Martin-Rabadan P, Munoz P, Fernandez-Cruz A, Bouza E. Prospective, randomised study of selective versus routine culture of vascular catheter tips: patient outcome, antibiotic use and laboratory workload. J Hosp Infect 2011;77:309-315.

6. Ekkelenkamp $\mathrm{MB}$, van der Bruggen $\mathrm{T}$, van de Vijver DA, Wolfs TF, Bonten MJ. Bacteremic complications of intravascular catheters colonized with Staphylococcus aureus. Clin Infect Dis 2008;46:114-118.

\section{Intensive Care Unit Probiotic Utilization Rates: When Committee Recommendations and Physician Utilization Diverge}

To the Editor-The intensive care units (ICUs) in most hospitals are high-risk settings for hospital-acquired diarrhea. Patients in the ICU are likely to have numerous comorbidities, to be of older age, and to have concomitant antibiotic use-all major risk factors for Clostridium difficile infection (CDI). ${ }^{1}$ Human gut flora is composed of trillions of microbes working in a symbiotic relationship with the human immune system to prevent colonization of opportunistic bacteria, often occurring with antibiotic usage and other illnesses. Probiotics, or oral preparations of live microorganisms, can stabilize the gut flora and might prevent $\mathrm{CDI}{ }^{2,3}$ Though multiple studies and metaanalyses have demonstrated the efficacy of probiotics toward CDI primary prevention, ${ }^{2,4-6}$ guidelines of major societies, such as the American College of Gastroenterology (ACG), the Society for Healthcare Epidemiology of America (SHEA), and the Infectious Disease Society of America (IDSA), have not formally recommended probiotic use for primary prevention of CDI in any setting or for any patient demographic. ${ }^{7,8}$ Although recent evidence has suggested that probiotics administered close to 